\title{
Quantum turbulent velocity statistics and quasiclassical limit
}

\author{
A.W. Baggaley and C.F. Barenghi \\ School of Mathematics and Statistics, Newcastle University, \\ Newcastle upon Tyne, NE1 $7 R U$, United Kingdom
}

\begin{abstract}
Two research groups have measured turbulent velocity statistics in superfluid helium using different techniques. The results were in conflict: one experiment revealed Gaussian distributions (as observed in ordinary turbulence), the other experiment determined power-laws. To solve the apparent puzzle, we numerically model quantum turbulence as a tangle of vortex filaments, and conclude that there is no contradiction between the two experiments. The transition from Gaussian to power-law arises from the different length scales which are probed using the two techniques. We find that the average distance between the quantum vortices marks the separation between quantum and quasi-classical length scales.
\end{abstract}

Turbulence in superfluid helium $\left({ }^{4} \mathrm{He}\right)$ [1, 2] consists of a tangle of discrete, thin vortex filaments, each carrying one quantum of circulation $\kappa=h / m$ (where $h$ is Planck's constant and $m$ the mass of one atom). This 'quantum turbulence' [3, 4] is also studied in superfluid ${ }^{3} \mathrm{He}[5,[6]$ and in atomic Bose-Einstein condensates [7]. The absence of viscosity and the discrete nature of the vorticity distinguish quantum turbulence from ordinary (classical) turbulence. Nevertheless, experiments have also shown remarkable similarities: the same pressure drop along and pipes [8], drag on a moving body [9], vorticity decay [10], and distribution of kinetic energy over the length scales (Kolmogorov energy spectrum) [11, 12]. The relation between quantum turbulence and classical turbulence has thus become the focus of attention.

This report is concerned with the statistical properties of the components of the turbulent superfluid velocity field $\mathbf{v}$. In ordinary turbulence, experiments 13 ] and numerical simulations [14, 15] confirm that velocity statistics are Gaussian. The usual interpretation of this property is the following [16]. At any time $t$, the velocity field $\mathbf{v}$ at the point $\mathbf{r}$ is determined by the vorticity field $\boldsymbol{\omega}=\nabla \times \mathbf{v}$ via the Biot-Savart law [17]

$$
\mathbf{v}(\mathbf{r}, t)=\frac{1}{4 \pi} \int \frac{\boldsymbol{\omega}\left(\mathbf{r}^{\prime}, t\right) \times\left(\mathbf{r}-\mathbf{r}^{\prime}\right)}{\left|\mathbf{r}-\mathbf{r}^{\prime}\right|^{3}} d \mathbf{r}^{\prime} .
$$

Thus, if the point $\mathbf{r}$ is surrounded by many, randomly oriented vortical structures, Gaussianity results from the application of the Central Limit Theorem.

In the last few years, the development of particle tracking visualization techniques suitable for liquid helium [18, 19] has opened the way to the measurement of quantum turbulence statistics. Using solid hydrogen tracers, Paoletti et al. 20] at the University of Maryland deduced that, in superfluid helium, the velocity components of the turbulent velocity follow power-law distributions. Large velocity fluctuations are thus relatively more frequent than in ordinary turbulence, where these distributions are Gaussian [21]. The authors suggested that power-laws distributions arise from vortex reconnections, clearly high-velocity events.
The experiment of Paoletti et al. motivated theoretical work on the problem. White et al. [22] solved numerically the Gross-Pitaevskii equation for a Bose-Einstein condensate and determined velocity distributions in a variety of quantum vortex systems at zero temperature: twodimensional and three-dimensional trapped atomic condensates, three-dimensional homogeneous condensates, Onsager's two-dimensional vortex gas. In all systems, they found power-law distributions, in agreement with Paoletti et al. 20], even in the absence of vortex reconnections. Using results from previous work by Min and Leonard 23] on the numerical analysis of vortex methods (later confirmed by Weiss et al. [24]), White et al. 22.] concluded that the non-Gaussianity arises from the singular nature of the quantum vorticity, which causes an anomalously slow convergence to Gaussian behaviour.

Further theoretical work at non-zero temperatures seemed to strengthen this conclusion. Baggaley and Barenghi 25] used the vortex filament model to numerically study intense vortex tangles. They confirmed the Kolmogorov spectrum and the power-laws distributions of the velocity components. The vortex filament model was also used by Adachi and Tsubota 26], who found power-law distributions in counterflow turbulence (quantum turbulence driven by a heat flow).

In a recent experiment, Salort et al. 27] at CNRS Grenoble measured the turbulent velocity in a superfluid wind tunnel using a small Pitot tube. They confirmed the Kolmogorov spectrum, but reported Gaussian velocity distributions, in apparent contradiction with Paoletti et al. 20] and with the numerical simulations [22, 25, 26].

To solve this puzzle, firstly we consider the size of the probe relative to the distance between vortices in the two experiments. The radius $a$ of the tracer particles used by Paoletti et al. was $a \approx 10^{-4} \mathrm{~cm}$. The turbulence was created by a heat flux $\dot{Q}$ which drove a counterflow current [28] $v_{n s}=\left|v_{n}-v_{s}\right|=\dot{Q} /\left(\rho_{s} S T\right)$ between superfluid and normal fluid velocity components $v_{n}$ and $v_{s}$, where $\rho_{s}$ is the superfluid density and $S$ the specific entropy. For example, at $T=1.9 \mathrm{~K}$ the largest heat flux was $\dot{Q}=0.17 \mathrm{~W} / \mathrm{cm}^{2}$, corresponding to $v_{n s}=1.5 \mathrm{~cm} / \mathrm{s}$, with $\rho_{s}=0.0831 \mathrm{~g} / \mathrm{cm}^{3}$ and $S=0.709 \mathrm{~J} /(\mathrm{gK})$. Using 
the relation $L=\gamma^{2} v_{n s}^{2}$ with $\gamma \approx 140 \mathrm{~s} / \mathrm{cm}^{2}$ as reported in Ref. [28], one finds $\ell \approx 4.7 \times 10^{-3} \mathrm{~cm}$, where $L$ is the vortex line density (vortex length per unit volume) and $\ell \approx L^{-1 / 2}$. Paoletti et al. 20] took their velocity measurements during the decay of the turbulence, after switching off the heater, so the actual inter-vortex distance must have been larger than the above value of $\ell$. We conclude that, in the experiment of Paoletti et al. [20], $a / \ell \ll 1$; this means that, although the tracer particles must have altered in some measure the motion of the vortex lines, the velocity field was probed at very small length scales, between $\ell$ and $a$.

In the experiment of Salort et al. 27], the nozzle of the Pitot tube had diameter $0.06 \mathrm{~cm}$. The authors estimated 29] that the inter-vortex separation was between $\ell \approx 5 \times 10^{-4}$ and $20 \times 10^{-4} \mathrm{~cm}$. We conclude that in this experiment $a / \ell \gg 1$ (even without considering that the effective resolution in the streamwise direction, set by time dynamics of the probe, was perhaps much larger than $a[29]$ ). In summary, the experiment of Paoletti et al. 20] probed the velocity field between vortices, at length scales less than $\ell$, whereas the experiment of Salort et al. 27] detected motion at scales larger than $\ell$, containing many vortex lines.

Secondly, we perform the following numerical calculation. In both experiments the length scales involved were much larger than the vortex core radius, $a_{0}=$ $10^{-8} \mathrm{~cm}$, so it is appropriate to use the vortex filament model [30]. We assume that the vorticity is concentrated in space curves $\mathbf{s}(\xi, t)$ around which the circulation is $\kappa=10^{-3} \mathrm{~cm}^{2} / \mathrm{s}$. Eq. (11) reduces to

$$
\frac{d \mathbf{s}}{d t}=-\frac{\kappa}{4 \pi} \oint_{\mathcal{L}} \frac{(\mathbf{s}-\mathbf{r})}{|\mathbf{s}-\mathbf{r}|^{3}} \times \mathbf{d r},
$$

where the line integral extends over all vortex lines. The numerical techniques to discretize the vortex lines, to compute the time evolution, to de-singularize the BiotSavart integral and to algorithmically perform reconnections when two vortex lines become close to each other are described in the literature [26, 30] or in our previous papers [25, 31]. The computational domain is a periodic box of size $D=0.075 \mathrm{~cm}$. The distance between discretization points along the vortex filaments is held at approximately $\delta / 2$ where $\delta=0.001 \mathrm{~cm}$. To speedup the evaluation of Biot-Savart integrals we use a tree method [32] with critical opening angle $\theta=0.4$. For the sake of simplicity, we assume that the temperature is $T=0$. This means that no external forcing is needed to sustain the turbulence, because the total kinetic energy is conserved during the evolution (at least for the time scale of interest here; the finite discretization and the reconnection algorithm introduce small energy losses which model phonon emission as described in Ref. 31] ).

The initial condition at time $t=0$ consists of 100 straight, randomly oriented, vortex filaments discretized

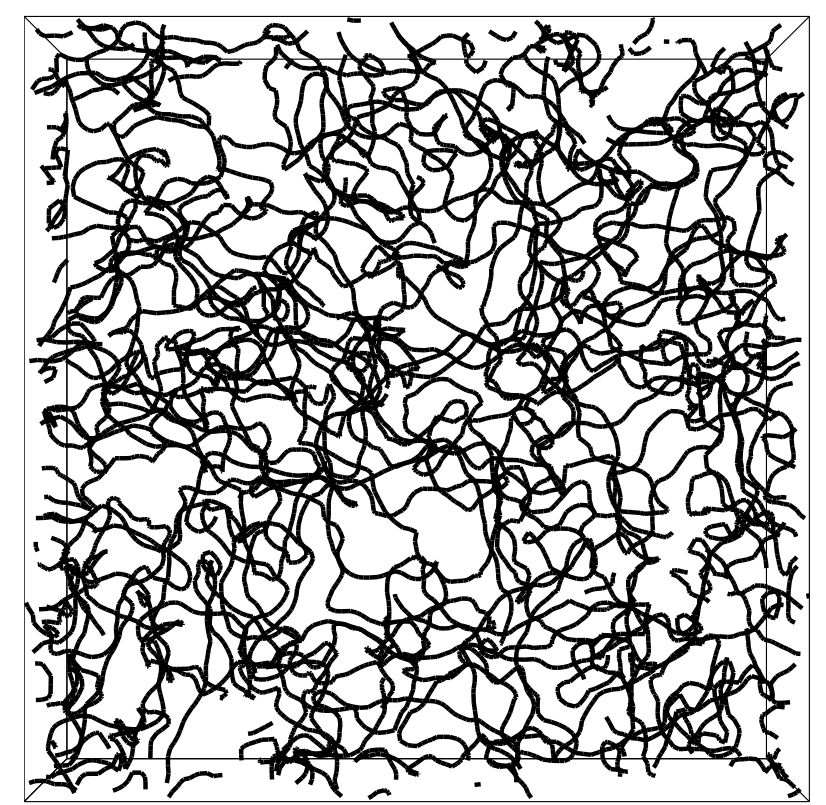

FIG. 1. Snapshot of the turbulent vortex tangle at $t=0.1 \mathrm{~s}$ after the vortex line density has saturated, the box size $D=$ $0.075 \mathrm{~cm}$.

over $N=10^{4}$ Lagrangian points $\mathbf{s}_{j}(j=1, \cdots N)$. During the evolution the vortex lines interact, become curved, reconnect, and a vortex tangle is quickly formed. We find that the vortex line density $L$ initially grows, then saturates to the value $L \approx 2.04 \times 10^{4} \mathrm{~cm}^{-2}$. We check that the average curvature saturates too. Fig. 1 shows the vortex tangle when we stop the calculation at $t=0.1 \mathrm{~s}$. We examine the energy spectrum $E(k)$, defined by

$$
E=\frac{1}{V} \int \frac{1}{2}|\mathbf{v}|^{2} d V=\int_{0}^{\infty} E(k) d k,
$$

where $E$ is the total energy per unit mass. Fig. 2 shows that most of the energy is contained in the large scales, and that the spectrum is qualitatively consistent with the Kolmogorov scaling $E_{k} \sim k^{-5 / 3}$ in the region $k \ll 2 \pi / \ell \approx 900 \mathrm{~cm}^{-2}$, in agreement with experiments [1, 12] and with previous numerical calculations [25, 3335]. The shallow spectrum at large values of $k$ is also consistent with existing similar calculations [34]. Secondly, we examine the velocity components and compute their probability density functions (normalized histograms, or PDF for short). Rather than sampling the values at points of the domain, we compute averages over regions of size $\Delta$, varying $\Delta$. This is done by calculating the velocity field, induced by the vortices, on a $128^{3}$ Cartesian mesh, and then averaging cells over the appropriate length scale. The result is shown in Fig. 3, where we compare the data against their Gaussian fits

$$
\operatorname{gPDF}\left(v_{i}\right)=\frac{1}{\sqrt{2 \pi \sigma^{2}}} \exp \left(-\left(v_{i}-\mu\right)^{2} /\left(2 \sigma^{2}\right)\right),
$$




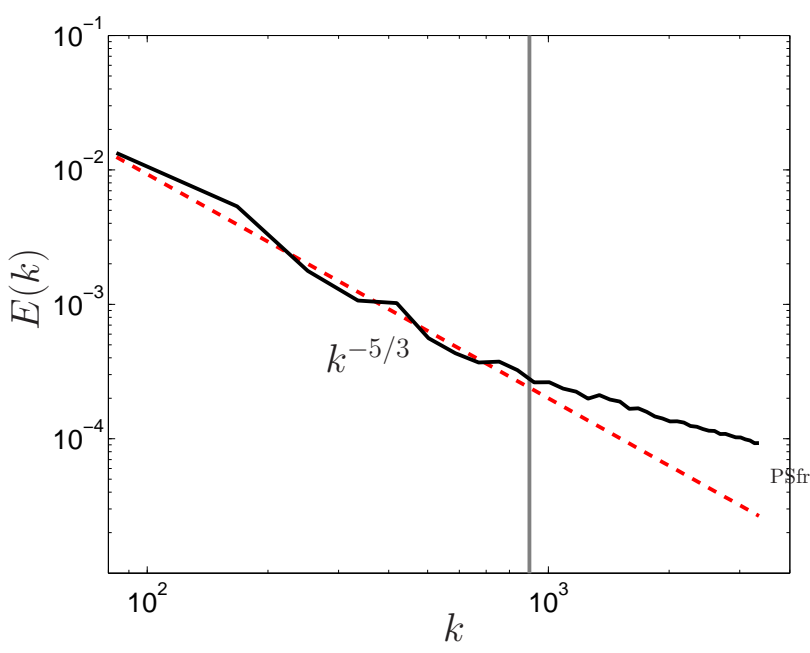

FIG. 2. (Color online) Energy spectrum $E_{k}$ (arbitrary units) vs wavenumber $k\left(\mathrm{~cm}^{-1}\right)$ corresponding to Fig. 1. The dashed line is the Kolmogorov scaling $E_{k} \sim k^{-5 / 3}$; the solid grey line shows wavenumber corresponding to the intervortex spacing $k_{\ell} \approx 900 \mathrm{~cm}^{-1}$.

where $\mu \approx 0$ and $\sigma$ is the standard deviation. It is apparent that, if $\Delta<\ell$, the PDFs are not Gaussian; by fitting $\operatorname{PDF}\left(v_{i}\right) \sim v_{i}^{-b}$ we obtain $b=3.2,3.2$ and 3.1 for $i=x, y, z$ respectively, in agreement with the power-law statistics found experimentally by Paoletti et al. 20] and numerically by White et al [22], Baggaley and Barenghi 25], and Adachi and Tsubota [26]. If $\Delta>\ell$, however, the distributions are Gaussian, and we recover the same (classical) statistics measured by Salort et al. [27].

In conclusion, the different velocity statistics observed by the Maryland and Grenoble experiments are consistent with each other. Taken together, these experiment and this work support the interpretation that, at scales larger than $\ell$, quantum turbulence exhibits quasi-classical behaviour (in terms of both energy spectrum and velocity statistics), whereas at scales smaller than $\ell$ (but still orders of magnitude larger than the quantum coherence length $a_{0}$ ), the discrete nature of quantized vorticity affects the distribution of energy over the length scales and the frequency of high-velocity events.

We thank J. Salort and P.E. Roche for providing us with experimental data and comments in advance of publication. This work was funded by the HPC-EUROPA2 project 228398, with the support of the European Community (Research Infrastructure Action of the FP7), and by the Leverhulme Trust (Grants F/00125/AH and RPG097).
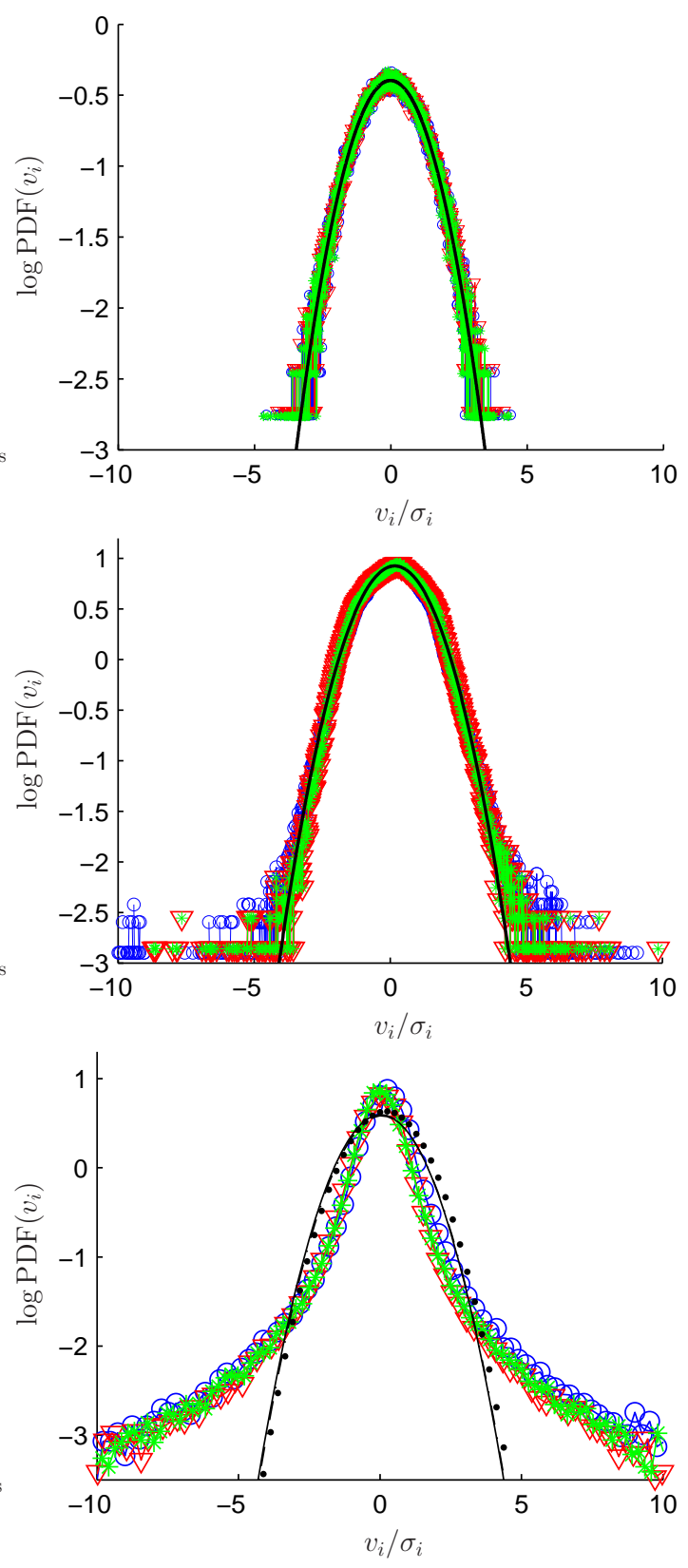

FIG. 3. (Color online) Probability density functions (PDF) of turbulent velocity components $v_{i}(i=x, y, z)$ vs $v_{i} / \sigma_{i}$ calculated by averaging over regions of size $\Delta=2 \ell$ (top), $\Delta=\ell$ (middle) and $\Delta=\ell / 6$ (bottom). (Green) asterisks, (blue) circles and (red) triangles refer respectively to $i=x, i=y$ and $i=z$ components. The solid lines ares the Gaussian fits, defined by Eq. 4 where $\bar{\sigma}=0.041$ (top), 0.048 (middle) and $0.082 \mathrm{~cm} / \mathrm{s}$ (bottom) respectively.

[1] P.M. Walmsley, and A.I. Golov, Quantum and quasiclassical types of superfluid turbulence, Phys. Rev. Lett. 100, 245301 (2008).

[2] T.V. Chagovets, and L. Skrbek, Steady and decaying flow 
of HeII in a channel with ends blocked by superleaks, Phys. Rev. Lett. 100, 2153 (2008).

[3] W.F. Vinen, and J.J. Niemela, Quantum turbulence. J. Low Temp. Phys. 128, 167 (2002).

[4] B. Halperin, and M. Tsubota (eds.), Quantum Turbulence, Progress in Low Temperature Physics, Vol. 16, Elsevier (2008).

[5] D.I. Bradley et al., Direct measurement of the energy dissipated by quantum turbulence, Nature Physics 7, 473 (2011).

[6] V.B. Eltsov et al., Quantum turbulence in a propagating superfluid vortex front, Phys. Rev. Lett. 99: 265301 (2007).

[7] E.A.L. Henn, J.A. Seman, K.M.F. Mugalhaes, and V.S. Bagnato, Emergence of turbulence in an oscillating BoseEinstein condensate, Phys. Rev. Lett. 103, 045301 (009).

[8] P.L. Walstrom, J.G. weisend, J.R. Maddocks, and S.W. Van Sciver, Turbulent flow pressure drop in various He II transfer system components, Cryogenics 28, 101 (1988).

[9] M.R. Smith, D.K. Hilton, and S.W. Van Sciver, Observed drag crisis on a sphere in flowing He I and He II. Phys Fluids 11, 751 (1999).

[10] S.R. Stalp, L. Skrbek, and R.J. Donnelly, Decay of grid turbulence in a finite channel, Phys. Rev. Lett. 82, 4831 (1999).

[11] J. Maurer, and P. Tabeling. Local investigation of superfluid turbulence. Europhys Lett 43, 29 (1998).

[12] J. Salort et al., Turbulent velocity spectra in superfluid flows. Phys Fluids 22, 125102 (2010).

[13] A. Noullez, G. Wallace, W. Lempert, R.B. Miles and U. Frisch, Transverse velocity increments in turbulent flow using the RELIEF technique, J. Fluid Mech. 339, 287 (1997).

[14] A. Vincent, and M. Meneguzzi, The spatial structure and statistical properties of homogeneous turbulence, J. Fluids Mech. 225, 1 (1991).

[15] T. Gotoh et al., D. Fukayama and T. Nakano, Velocity field statistics in homogeneous steady turbulence obtained using a high-resolution direct numerical simulation, Phys. of Fluids 14, 1065 (2002).

[16] P.A. Davidson, Turbulence, Oxford University Press (2004).

[17] P.G. Saffman, Vortex Dynamics, Cambridge University Press, Cambridge (1992). '

[18] T. Zhang, and S.W. Van Sciver, Large-scale turbulent flow arounbd a cylinder in counterflow superfluid ${ }^{4} \mathrm{He}$ (He II), Nature Physics 1, 36 (2005).

[19] G.P. Bewley, D.P. Lathrop, and K.R. Sreenivasan, Superfluid helium: visualization of quantized vortices, Nature 441, 588 (2006).

[20] M.S. Paoletti, M.E. Fisher, K.R. Sreenivasan, and D.P. Lathrop, Velocity statistics distinguish quantum turbu- lence from classical turbulence, Phys. Rev. Lett. 101, 154501 (2008).

[21] The Gaussianity of the distributions of the components of $\mathbf{v}$ should not be confused with the non-Gaussianity of the distributions of the gradients of $\mathbf{v}$, or of the joint probability distribution of $\mathbf{v}$ at two points, which are usually observed in ordinary turbulence.

[22] A.C. White et al., Nonclassical velocity statistics in a turbulent atomic Bose-Einstein condensate, Phys. Rev. Lett. 104, 075301 (2010).

[23] I.A. Min, I. Mezic, and A. Leonard, Lévy stable distributions for velocity and velocity difference in systems of vortex elements, Phys. Fluids 8, 1169 (1996).

[24] J.B. Weiss, A. Provenzale, and J. C. McWilliams, Lagrangian dynamics in high-dimensional point-vortex systems, Phys. Fluids 10, 1929 (1998).

[25] A.W. Baggaley, and C.F. Barenghi, Vortex-density fluctuations in quantum turbulence, Phys. Rev. B 84, 020504(R) (2011).

[26] Hi. Adachi, and M. Tsubota, Numerical study of velocity statistics in steady counterflow quantum turbulence, Phys. Rev. B 83, 132503 (2011).

[27] J. Salort, B. Chabaud, E. Lévêque and P.-E. Roche, Energy cascade and the four-fifths law in superfluid turbulence, HAL-00635578 (2011).

[28] H. Adachi, S. Fujiyama, and M. Tsubota, Steady-state counterflow quantum turbulence. Simulation of vortex filaments using the full Biot-Savart law. Phys. Rev. B 81, 104511 (2010).

[29] P.E. Roche, private communication.

[30] K.W. Schwarz, Three-dimensional vortex dynamics in superfluid ${ }^{4}$ He: Homogeneous superfluid turbulence, Phys Rev B 38, 2398 (1988).

[31] A.W. Baggaley, and C.F. Barenghi, Spectrum of turbulent Kelvin-waves cascade in superfluid helium, Phys. Rev. B 83, 134509 (2011).

[32] A.W. Baggaley, and C.F. Barenghi, Tree method for quantum vortex dynamics, arXiv:1108.1119, to be published in J. Low Temp. Phys.

[33] C. Nore, M. Abid, and M.E. Brachet, Kolmogorov turbulence in low-temperature superflows, Phys. Rev. Lett. 783896 (1997).

[34] T. Araki, M. Tsubota, and S.K. Nemirovskii, Energy spectrum of superfluid turbulence with no normal-fluid component. Phys. Rev. Lett. 89145301 (2002).

[35] M. Kobayashi, and M. Tsubota, Kolmogorov spectrum of superfluid turbulence: numerical analysis of the GrossPitaevskii equation with a small-scale dissipation, Phys. Rev. Lett. 94065302 (2005).

[36] V.S. L'vov, S.V. Nazarenko, and L. Skrbek, Energy spectra of developed turbulence in helium superfluids, J. Low Temp. Phys. 145125 (2006). 